\title{
VIABILIDADE DE PÓLEN EM CLONES DE LARANJA PÊRA E OUTRAS VARIEDADES ASSEMELHADAS ${ }^{1}$
}

\author{
POLLEN VIABILITY OF CV. 'PERA' CLONES AND OTHER \\ SWEET ORANGE VARIETIES
}

\author{
Edson Tobias Domingues ${ }^{2,3}$, Augusto Tulmann Neto ${ }^{4}$, Joaquim Teófilo Sobrinho ${ }^{2}$
}

\section{RESUMO}

A esterilidade é limitante para programas que envolvam a hibridação sexual, mas por outro lado tem importância econômica em citros por resultar em menor número de sementes por fruto em certas variedades. A variedade Pêra é a mais plantada no país, em torno de $50 \%$ do total, possui boa qualidade tanto para o processamento quanto para o consumo in natura. A importância desse cultivar para a citricultura nacional e a necessidade de se buscar novos cultivares, que combinem as boas características desse material com outras características desejáveis à cultura, enfatiza a necessidade de estudos básicos visando ao melhor conhecimento da genética e da reprodução dessa variedade. Nesse sentido, foram estudados quanto à viabilidade do pólen, onze clones de laranja Pêra e cinco variedades assemelhadas a esta, enxertados sobre limoeiro Cravo. O percentual de pólen viável foi avaliado através da coloração com carmim acético a $25 \%$ e contagem sob microscópio ótico. As variedades Corsa Tardia, Pêra Olímpia e Ovale San Lio apresentaram os maiores percentuais de pólen viável, com 65,5, 57,3 e 53,6\%, respectivamente, sendo que a maioria dos genótipos estudados apresentaram viabilidade do pólen variando de 30 a 50\%. Os genótipos que apresentaram menores valores de viabilidade foram a Pêra Bianchi e Pêra Pirangi, com aproximadamente $28 \%$ de pólen viável. Na variedade Ovale de Siracusa, praticamente não se observou presença de pólen.

Palavras-chave: Citrus sinensis [L.] Osbeck, melhoramento genético.

\section{SUMMARY}

Sterility limits sexual hybridization, but on the other hand, it is of economic importance by inducing fewer seeds per fruit in some citrus varieties. Pera sweet orange is the main

\begin{abstract}
cultivar in Brazil, around 50\% of all sweet oranges produced in the country. This variety has good processing quality as well as for the fresh market. The importance of this cultivar for the brazilian citriculture and the necessity to search for new cultivars, which combine the good characteristics from Pera with others desirable agronomic characters, has induced new basic studies aiming at a better knowledge of genetics and reproduction of this elite variety. In this way, in this work, was studied the pollen viability for eleven clones of Pera and five others sweet orange varieties, morphologically similar to Pera, budded in Rangpur lime (Citrus limonia Osbeck). The percentile of pollen viability was evaluated using acetic carmine stain and optic microscopy. It was observed that Corsa Tardia, Pera Olímpia and Ovale San Lio varieties, presented larger quantity of viable pollen, with 65.5, 57.3 and $53.6 \%$, respectively, most of studied genotypes showed this values varying from 30 to 50\%, and those that presented fewer values were Pera Bianchi and Pera Pirangi, with approximately $28 \%$ of viable pollen as well as Ovale de Siracusa variety, which did not present pollen.
\end{abstract}

Key words: Citrus sinensis [L] Osbeck, genetic breeding.

\section{INTRODUÇÃO}

O Brasil é o maior produtor mundial de laranjas, Citrus sinensis (L.) Osbeck, com 14,9 milhões de toneladas/ano no período de 1990 a 1996 (cerca de $25 \%$ do total mundial). O Estado de São Paulo foi responsável naquele período por $83 \%$ do total produzido no país, com 12,4 milhões de toneladas; explorando comercialmente s cultivares Pêra

\footnotetext{
${ }^{1}$ Parte da Tese defendida pelo primeiro autor para a obtenção de título de doutor em Genética e Melhoramento de Plantas da ESALQ/USP, Piracicaba (SP), em 02/02/1999.

${ }^{2}$ Engenheiro Agrônomo, Pesquisador Científico, Centro de Citricultura Sylvio Moreira - Instituto Agronômico de Campinas, Caixa Postal 04, 13470-970, Cordeirópolis, SP, Brasil. Autor para correspondência.

${ }^{3}$ Aluno de Doutorado, Bolsista CNPq, Departamento de Genética, Escola Superior de Agricultura Luiz de Queiroz/Universidade de São Pailo.

${ }^{4}$ Engenheiro Agrônomo, Pesquisador Científico, Centro de Energia Nuclear na Agricultura / USP. 
(47\%), Natal (26\%), Valência (14\%) e Hamlin (5\%), entre outras (7\%) (FAO, 1996; AMARO E MAIA, 1997). Apesar da grande importância da citricultura nacional e paulista, pode-se observar que o número de variedades exploradas é restrito, se comparado ao potencial existente em recursos genéticos para a variedade (DOMINGUES et $\boldsymbol{a l}$., 1995). Dessa maneira há diversos trabalhos buscando a obtenção de novos cultivares para ampliar a base genética dos pomares e, com isso, evitar possíveis colapsos da citricultura nacional.

Os principais problemas encontrados para a produção atual e futura dos citros no país são as doenças, como o declínio, a tristeza, a leprose, o cancro cítrico e a clorose variegada dos citros, o mais recente problema a trazer preocupação (DOMINGUES, 1997a). Entre as pragas da cultura temos os ácaros da ferrugem e leprose, cochonilhas, coleópteros, bicho furão, além da mais recente praga no país, a lagarta minadora dos citros. A elevação da produtividade, melhoria da qualidade dos frutos e redução do número de sementes por fruto e do período de entre-safra são outros aspectos visados para o melhoramento de variedades copa. Para os portaenxertos, busca-se variedades que sejam resistentes à Tristeza, Declínio, Gomose e a pragas como as cochonilhas, nematóides e as brocas, além de melhor compatibilidade desse porta-enxerto com as principais variedades copa e, se possível, que permitam a redução do porte dessas copas, o que facilitaria o manejo, a colheita e o plantio mais adensado.

Apesar da importância dessa cultura, muito pouco tem sido estudado dos aspectos genéticos básicos para o melhoramento dessa espécie e a maioria dos problemas citados certamente encontrarão solução eficaz através do cultivo adequado dos atuais cultivares somados a novos, a serem obtidos através de cruzamentos orientados. Para o melhoramento genético, no entanto, deve-se partir dos melhores cultivares da citricultura nacional para garantir a aceitação dos futuros híbridos, apesar da elevada heterozigosidade existente na espécie. Devido a grande importância comercial da variedade Pêra, tanto para o mercado interno de frutas frescas como para o processamento industrial, torna-se fundamental uma caracterização genética mais detalhada da variedade e de seus clones variantes, originados por mutações somáticas ou zigóticas ocorridas nessa variedade, bem como das variedades que apresentem características semelhantes. Nesse sentido, diferentes trabalhos têm sido realizados visando a quantificar diferenças de produtividade (TEÓFILO SOBRINHO et al. 1992), de grau de poliembrionia (DOMINGUES et al. 1997d) e de maturação, e de qualidade de fruta (DOMINGUES et al. 1996, 1997b).
Uma vez que a análise da fertilidade do pólen é condição preliminar indispensável ao melhoramento genético, o presente trabalho objetivou determinar a viabilidade do pólen de clones de laranjeira Pêra e variedades assemelhadas para programas futuros de hibridação sexual envolvendo estes genótipos. Esse é um importante passo no estudo da citada auto-incompatibilidade presente na espécie Citrus sinensis [L] Osbeck, produtora de laranjas doces (SOOST \& CAMERON, 1975), bem como dos fatores que levam à esterilidade do pólen.

\section{MATERIAL E MÉTODOS}

Os dados foram coletados em um experimento instalado em dezembro de 1980 na então Estação Experimental Sylvio Moreira do Instituto Agronômico do Estado de São Paulo, hoje Centro de Citricultura Sylvio Moreira, situado em Cordeirópolis, SP, (CCSM/IAC). Para estudo da viabilidade de pólen foram utilizados 11 clones de laranjeira Pêra (Bianchi, Dibbern, EEL, GS - 2000, Olímpia, Premunizada - 1212, Premunizada - 1743, R. Gullo 1569, R. Gullo - 1570, Pirangi e Vimusa) e as variedades Ovale, Ovale de Siracusa, Ovali San Lio, Corsa Tardia e Lamb's Summer, produtoras de frutos com aspectos morfológicos semelhantes aos da laranjeira Pêra. $\mathrm{O}$ experimento foi implantado no delineamento de blocos casualizados com 6 repetições, em espaçamento de $7 \mathrm{~m} \times 5 \mathrm{~m}$, sendo o portaenxerto o limoeiro Cravo (Citrus limonia Osbeck). O solo da área experimental é um Latossolo vermelho-escuro distrófico e o clima da região é do tipo Cwa. O experimento foi conduzido sem irrigação e se encontrava no $12^{\underline{0}}$ ano de produção (ano agrícola de 1995). Foram coletados 12 botões florais em máximo desenvolvimento, antes da antese. Os botões foram mantidos em placas de Petri até a abertura floral, o que levou aproximadamente 24h. Após a abertura dos botões, as anteras foram coletadas, com o auxílio de pinças e armazenadas em placas de Petri até a deiscência e liberação do pólen, o qual foi acondicionado em tubos 'ependorf', coberto com solução 3:1 de álcool absoluto e ácido acético e mantido à temperatura ambiente. Após 24 horas, esses tubos foram mantidos em geladeira a, aproximadamente, $4^{\circ} \mathrm{C}$.

Os estudos sobre a viabilidade do pólen foram feitos no Laboratório da Seção de Radiogenética do CENA/USP, através da coloração dos grãos de pólen com carmim acético a $25 \%$ e da contagem dos grãos de pólen viáveis e não viáveis, sob microscópio ótico, seguindo metodologia apresentada por MOREIRA \& GURGEL (1940 e 1941). Foram calculados os percentuais de pólen viável por varie- 
dade. Foi realizada a análise de variância [percentuais calculados, transformados segundo arco seno $\sqrt{ }(\mathrm{x} / 100)]$. As médias foram contrastadas pelo teste de Tukey em nível de 5\% de probabilidade. Foram estimadas as correlações (produto momento de Pearson, para $(\mathrm{p}<0,05)$ entre o percentual de pólen viável com a produção média de frutos por planta, utilizando dados obtidos por TEÓFILO SOBRINHO et al. (1992), e DOMINGUES et al. (1997b), com número médio de sementes por fruto e com o percentual de poliembrionia obtido pela contagem dos embriões nas sementes (utilizando dados obtidos por DOMINGUES et $\boldsymbol{a l} .$, 1997d) para os onze clones de laranja Pêra e para as cinco variedades estudadas.

\section{RESULTADOS E DISCUSSÕES}

O percentual de pólen funcional ou viável varia grandemente entre espécies e cultivares. Alguns dos cultivares mais amplamente utilizados são deficientes a esse respeito (SOOST \& CAMERON, 1975). No presente trabalho, foram observados, para os diferentes genótipos estudados, grãos de pólen: a) parcialmente coloridos e uniformes, considerados viáveis; b) meio coloridos que deixavam dúvida quanto à viabilidade; c) de tamanho normal mas vazios; d) grãos de pólen pequenos, aparentemente inviáveis e degenerados. MOREIRA \& GURGEL (1941) também observaram esses tipos de pólen, e após testes de germinação, concluíram que apenas os grãos de pólen bem coloridos e circulares apresentavam-se viáveis. Como no trabalho daqueles autores, neste foram separados os grãos de pólen avaliados em duas categorias, a dos viáveis (a) e a dos não viáveis (b, c e d). De um modo geral, os grãos de pólen viáveis apresentaram-se melhor desenvolvidos, mais circulares e coraram-se melhor na presença do carmin acético, já os inviáveis, apresentaramse mal formados, normalmente elípticos e pouco corados pelo carmin. MOREIRA \& GURGEL (1941) utilizaram carmin acético a $40 \%$ para coloração dos grãos de pólen, no presente trabalho; no entanto, notou-se que a concentração de $25 \%$ foi suficiente. Foi observado que mesmo concentrações mais baixas permitem boas análises da viabilidade do pólen e que as concentrações maiores do corante permitem a obtenção de fotos mais nítidas.

Observou-se que as variedades Corsa Tardia, Pêra Olímpia e Ovale San Lio apresentaram os maiores percentuais de pólen viável, com 65,5, 57,3 e 53,6\%, respectivamente. A maioria dos genótipos estudados apresentaram esse valor, variando de 30 a $50 \%$, e as que apresentaram menores valores foram a Pêra Bianchi e Pêra Pirangi, com aproximadamente $28 \%$ de pólen viável, além da variedade
Ovale de Siracusa, na qual, praticamente, não se observou presença de pólen (tabela 1).

MOREIRA \& GURGEL (1941) observaram que, embora existam diferenças de viabilidade de pólen para diferentes ramos da mesma planta, diferentes plantas da mesma variedade, plantas de diferentes origens, sobre diferentes porta-enxertos e em diferentes épocas, essas diferenças de um modo geral são pequenas. Esses autores notaram que entre genótipos é que são encontradas as maiores diferenças. Trabalharam com diferentes espécies de citros e encontraram grande variabilidade de resposta entre variedades, observaram, por exemplo, o nível de $10 \%$ de fertilidade para a variedade Perão e $96 \%$ para uma variedade de Cidra, encontraram nesse mesmo trabalho grande diferença de resposta entre variedades de laranjas doces.

Observou-se que mesmo entre clones selecionados, de uma mesma variedade, mas que apresentam níveis diferenciados de produtividade existiram diferenças no nível da fertilidade do pólen. Os clones estudados poderiam ser separados, aproximadamente, em três grupos quanto ao nível de fertilidade: o primeiro, composto dos clones Bianchi, Pirangi, Dibbern e Premunizado-1743, com valores crescentes, de 28 a $35 \%$ de pólen viável. O segundo, dos clones R.Gullo-1570, Premunizado-1212, Vimusa, R.Gullo-1569, GS-2000 e EEL com valores de 41 a $46 \%$ de pólen viável; e o terceiro grupo composto apenas pela Pêra Olímpia, com 57\% de pólen viável. MOREIRA \& GURGEL (1940 e 1941) obtiveram, para plantas de laranja Pêra, de diferentes origens, níveis de fertilidade do pólen variando de 36 a $49 \%$, sendo, portanto, concordante com a resposta da maioria dos clones aqui estudados.

Embora a diferença de viabilidade de pólen tenha sido perceptível mesmo entre clones de uma mesma variedade, as maiores amplitudes de variação realmente foram encontradas comparando-se diferentes variedades. A variedade Ovale de Siracusa praticamente não apresentou pólen nas anteras, enquanto que a 'Ovale' e 'Lamb's Summer' apresentaram 30\% de pólen viável; já a variedade Ovale San Lio apresentou 54\% e a Corsa Tardia $66 \%$.

MOREIRA \& GURGEL (1941) citam que, de um modo geral, variedades com elevado percentual de poliembrionia apresentavam também elevado percentual de pólen viável. Encontraram, ainda, correlação significativa mediana $(+0,50)$ entre o número de sementes e o percentual de pólen viável. No presente trabalho, buscou-se estudar as correlações entre o percentual de pólen viável com a produção média de frutos por planta de 1984 a 1991 (utilizando dados obtidos por TEÓFILO SOBRINHO et $\boldsymbol{a l}$., 1992, e DOMINGUES $\boldsymbol{e}$ t al., 
Tabela 1 - Percentual de pólen viável, produção média de 1984 a 1988, peso de 30 sementes, número de sementes por fruto, número de embriões observados por semente e taxa de poliembrionia correspondente, para 11 clones da variedade Pêra e 5 variedades assemelhadas. ${ }^{1}$.

\begin{tabular}{|c|c|c|c|c|c|}
\hline Variedade ou clone & $\begin{array}{l}\text { Percentual de } \\
\text { pólen viável }^{2}\end{array}$ & $\begin{array}{l}\text { Produção média de } \\
\text { frutos de 1984-1988 }\end{array}$ & $\begin{array}{c}\text { Peso de } 30 \\
\text { sementes }\end{array}$ & $\begin{array}{l}\text { Número de } \\
\text { sementes por } \\
\text { fruto }\end{array}$ & $\begin{array}{c}\text { N. embriões observados } \\
\text { por semente }\end{array}$ \\
\hline
\end{tabular}

\begin{tabular}{|c|c|c|c|c|c|c|}
\hline & $\%$ & $\mathrm{~kg} / \mathrm{planta}$ & $\mathrm{g}$ & & & $\%$ \\
\hline Pêra Vimusa & 43,6 cde & 58,4 & 3,9 & $3,7 \mathrm{abc}$ & $2,7 \mathrm{bcd}$ & $66,7 \mathrm{bcd}$ \\
\hline Pêra EEL & $45,6 \mathrm{bcd}$ & 64,3 & 3,0 & $2,9 \mathrm{bcd}$ & $1,4 \mathrm{~d}$ & $41,6 \mathrm{~d}$ \\
\hline Pêra GS-2000 & 43,7 cde & 80,0 & 4,2 & $2,9 \mathrm{bcd}$ & $3,4 \mathrm{bc}$ & $85,7 \mathrm{ab}$ \\
\hline Pêra Olímpia & $57,3 \mathrm{ab}$ & 41,4 & 4,3 & $5,7 \mathrm{ab}$ & $4,0 \mathrm{~b}$ & $83,3 \mathrm{ab}$ \\
\hline Pêra Premuniz.-1212 & 43,4 cde & 44,3 & 3,1 & $4,0 \mathrm{abc}$ & $6,2 \mathrm{a}$ & $100,0 \mathrm{a}$ \\
\hline Pêra Bianchi & $28,1 \mathrm{fg}$ & 64,4 & 4,3 & $4,8 \mathrm{abc}$ & $4,3 \mathrm{ab}$ & $85,7 \mathrm{ab}$ \\
\hline Pêra R.Gullo 1569 & 43,7 cde & 48,1 & 2,8 & $4,4 \mathrm{abc}$ & $2,0 \mathrm{~cd}$ & $50,0 \mathrm{~cd}$ \\
\hline Pêra Dibbern & $31,2 \mathrm{de}$ & 53,4 & 2,7 & 2,6 cde & $3,2 \mathrm{bc}$ & $80,0 \mathrm{abc}$ \\
\hline Pêra Pirangi & 28,4 efg & 34,0 & 3,7 & $7,3 \mathrm{a}$ & $4,3 \mathrm{ab}$ & $83,3^{\mathrm{a}} \mathrm{b}$ \\
\hline Pêra R.Gullo 1570 & 41,1 cde & 41,1 & 2,8 & $4,7 \mathrm{abc}$ & $4,2 \mathrm{ab}$ & $80,0 \mathrm{abc}$ \\
\hline Pêra Premuniz.-1743 & 34,7 de & 70,5 & 3,3 & $4,4 \mathrm{abc}$ & $3,2 \mathrm{bc}$ & $100,0 \mathrm{a}$ \\
\hline Ovale & $30,4 \mathrm{def}$ & 34,0 & 3,8 & $0,4 \mathrm{e}$ & $4,2 \mathrm{ab}$ & $60,0 \mathrm{bcd}$ \\
\hline Ovale de Siracusa & $-\mathrm{h}$ & 33,0 & 3,1 & $5,9 \mathrm{ab}$ & $4,0 \mathrm{~b}$ & $87,5 \mathrm{ab}$ \\
\hline Ovale San Lio & $53,6 \mathrm{abc}$ & 28,1 & 3,1 & $1,1 \mathrm{de}$ & $4,3 \mathrm{ab}$ & $100,0 \mathrm{a}$ \\
\hline Lamb's Summer & $30,2 \mathrm{def}$ & 59,1 & 4,9 & $4,9 \mathrm{abc}$ & $3,4 \mathrm{bc}$ & $66,7 \mathrm{bcd}$ \\
\hline Corsa Tardia & $65,5 \mathrm{a}$ & 57,7 & 3,6 & $1,1 \mathrm{de}$ & $3,3 \mathrm{bc}$ & $62,5 \mathrm{bcd}$ \\
\hline dms (Tukey, 5\%) & 8,97 & - & & 0,77 & 0,41 & 28,67 \\
\hline C.V. $(\%)$ & 22,67 & - & & 33,54 & 16,93 & 34,61 \\
\hline
\end{tabular}

${ }^{1}$ Médias seguidas de letras iguais não diferem significativamente entre si (sentido vertical) pelo teste de Tukey - 5\%. ${ }^{2}$ Dados obtidos no Laboratório de Radiogenética - CENA/USP. ${ }^{3}$ Segundo Teófilo Sobrinho et al. (1992) e Domingues et al. (1997b).

1997b), com o número médio de sementes por fruto e com o percentual de poliembrionia obtido pela contagem dos embriões nas sementes (utilizando dados obtidos por DOMINGUES et al., 1997d) para os mesmos genótipos do mesmo experimento aqui estudado (tabela 1). No entanto, as correlações encontradas foram pequenas e não significativas para os diferentes caracteres confrontados.

As características morfológicas do pólen são geneticamente estáveis e, portanto, úteis ao estudo da taxonomia de plantas. YIN-MIN et al. (1981) estudaram a taxonomia de 20 diferentes genótipos pertencentes a diferentes gêneros de citros e a diferentes espécies de Citrus, utilizando o estudo da morfologia do pólen sob microscópio eletrônico. Essa técnica também tem sido utilizada para estudos mais detalhados da biologia floral dessas espécies. CRESCIMMANO et al. (1988a e 1988b) estudaram, com esse intuito, diferentes genótipos de tangerinas e limões. No presente trabalho, foram observadas algumas diferenças de formato para os grãos de pólen viáveis dos diferentes genótipos, todavia, essas diferenças deverão ser quantificadas em estudos posteriores utilizando microscopia eletrônica.
Contudo, os resultados aqui apresentados contribuem para a caracterização de germoplasma dos citros como enfatizado nos descritores dos citros do IBPGR (1988).

Para utilização como parental masculino em programas de melhoramento, a maioria dos genótipos estudados mostrou-se adequada, exceto a variedade Ovale de Siracusa, a qual praticamente não produziu pólen. Já os clones Bianchi, Dibbern, Pirangi, Premunizado-1743, Ovale, e a variedade Lamb's Summer apresentaram em torno de $70 \%$ de pólen não viável e obrigariam, portanto, um número de repetições maior que as variedades com maior fertilidade de pólen, em programas de cruzamentos controlados. No entanto, existem referências indicando que a intensidade de pólen não funcional nos cultivares é, em geral, proporcional à intensidade de óvulos não funcionais, que abortam, sugerindo que as variedades com baixa fertilidade de pólen podem não ser bons parentais femininos. Algumas exceções ocorrem, como no caso das laranjas Bahia que, embora pólen-estéreis, produzem óvulos funcionais (SOOST \& CAMERON, 1975). Já a comprovação desses dados e a quantificação da fertilidade femini- 
na dos genótipos estudados no presente trabalho ficam prejudicadas pela elevada poliembrionia observada nos mesmos (DOMINGUES et al. 1997d).

\section{CONCLUSÃO}

Existe variabilidade no percentual de pólen viável para os diferentes clones de laranja Pêra.

Não existe correlação entre o percentual de pólen viável com a produção de frutos por planta, nem com o número de sementes produzidos por fruto ou o grau de poliembrionia observado nas sementes dos diferentes genótipos de laranja Pêra.

Para utilização como parental masculino em programas de melhoramento, a maioria dos genótipos é adequada, exceto a variedade Ovale de Siracusa, a qual praticamente não produz pólen.

\section{AGRADECIMENTOS}

À Secretaria de Agricultura e Abastecimento do Estado de São Paulo, ao CNPq e FUNDECITRUS pela colaboração no desenvolvimento desta pesquisa. Às estudantes Bruna Gardenal Fina, Viviana Yoshie Sugahara e Luciana A. Oliveira, pelo auxílio na coleta de material em campo e à colega Janay A. Santos pelas sugestões apresentadas para a coloração das amostras.

\section{REFERÊNCIAS BIBLIOGRÁFICAS}

AMARO, A.A., MAIA, M.L. Produção e comércio de laranja e de suco no Brasil. Laranja, v. 18, n. 1, p. 1-26, 1997.

CRESCIMMANO, F.G., PASQUALE, F., GERMANA, M.A., $\boldsymbol{e} t$ al. Ultrastrutural and physiological observations on pollen of five clementine (C. clementina Hort. ex. Tan.). In: SIXTH INTERNATIONAL CITRUS CONGRESS, Tel-Aviv, 1988a Proceedings... Weikrsheim: Margraf Scientific Books, 1988. 1850 p. p. $443-450$

CRESCIMMANO, F.G., PASQUALE, F., GERMANA, M.A., $\boldsymbol{e}$ al. Ultrastrutural and physiological observations on the pollen of six lemon cultivars (C. limon (1.) Burm. F.). In: SIXTH INTERNATIONAL CITRUS CONGRESS, Tel-Aviv, 1988a. Proceedings... Weikrsheim: Margraf Scientific Books, 1988. 1850 p. p. $451-457$
DOMINGUES, E.T. Bancos de germoplasma contribuem para a melhoria dos pomares. Informativo Agropecuário Coopercitrus, n. 124, p. 22-23, 1997a.

DOMINGUES, E.T., POMPEU JUNIOR, J., MACHADO, M.A., et al. Acessos mantidos no banco ativo de germoplasma de citros do Centro de Citricultura Sylvio Moreira/IAC. Cordeirópolis, SP: CCSM/IAC, 1995. 63 p. (Mimeografado).

DOMINGUES, E.T., TEÓFILO SOBRINHO, J., FIGUEIREDO, J.O., et al. . Qualidade e maturação das laranjas Ovale, Ovale de Siracusa e Ovale San Lio. Laranja, Cordeirópolis, v. 17, n. 1, p. 143-158, 1996.

DOMINGUES, E.T., TEÓFILO SOBRINHO, J., POMPEU JUNIOR, J., et al. Caracterização do potencial agronômico das laranjeiras Redonda e Corsa Tardia. Revista Brasileira de Fruticultura, v. 19, n. 1, 1997b. (no prelo).

DOMINGUES, E.T., TULMANN NETO, A., TEÓFILO SOBRINHO, J. et al. Polyembryony study for clones of Pera and others sweet orange varieties. Revista Brasileira de Genética, Ribeirão Preto, v. 20, n. 3, supplement, p. 147, 1997 d.

FAO. Interamerican citrus network. Newsletter. Campinas: IACNET), 1996. n. 11, 24 p.

IBPGR. Descriptors for Citrus. Rome: International Board for Plant Genetic Resources, 1988. 27 p.

MOREIRA, S., GURGEL, J.T.A. A fertilidade do pólen e sua correlação com o número de sementes, em espécies e formas do gênero Citrus. Bragantia, Campinas, v. 1, p. 669-711, 1941.

MOREIRA, S., GURGEL, J.T.A. Relatório da Seção de Citricultura. Campinas: IAE, 1940. Exame e contagem de pólen: p. 53-62.

SOOST, R.K., CAMERON, J.W. Citrus. In: JANICK J., MOORE, J.N. (eds.). Advances in Fruit Breeding. West Lafayette, Indiana: Purdue University, 1975. p. 507-540.

TEÓFILO SOBRINHO, J., POMPEU JUNIOR, J., FIGUEIREDO, J. O. de, et al. Competição entre quinze clones de laranja pêra na região de Limeira. Revista Brasileira de Fruticultura, Cruz das Almas, v. 14, n. 1, p. 41-48, 1992.

YIN-MIN, Y., YAN, K., XIANG-HONG, Z Studies on pollen morphology of citrus plants. Proc Int Soc Citriculture, n. 1, p. 23-25, 1981. 